\title{
THE PRECESSION OF THE HERBIG-HARO 111 FLOW IN THE INFRARED
}

\author{
A. Noriega-Crespo ${ }^{1}$, A. C. Raga ${ }^{2}$, V. Lora ${ }^{3}$, K. R. StapelfeldT ${ }^{4}$, And S. J. Carey ${ }^{1}$ \\ ${ }^{1}$ Spitzer Science Center, California Institute of Technology, CA 91125, USA \\ ${ }^{2}$ Instituto de Ciencias Nucleares, Universidad Nacional Autónoma de México, Ap. 70-543, 04510 D. F., Mexico \\ ${ }^{3}$ Astronomisches Rechen-Institut Zentrum für Astronomie der Universität Heidelberg, Mönchhofstr, 12-14 69120 Heidelberg, Germany \\ ${ }^{4}$ Jet propulsion Laboratory, California Institute of Technology, MS 183-900, 4800 Oak Grove Drive, Pasadena, CA 91109, USA \\ Received 2011 February 11; accepted 2011 March 29; published 2011 April 11
}

\begin{abstract}
We present Spitzer Infrared Array Camera images of the Herbig-Haro (HH) 111 outflow that show a wealth of condensations/knots in both jet and counterjet. Studying the positional distribution of these knots, we find very suggestive evidence of a mirror symmetric pattern in the jet/counterjet flow. We model this pattern as the result of an orbital motion of the jet source around a binary companion. From a fit of an analytic, ballistic model to the observed path of the HH 111 system, we find that the motion in a binary with two $\sim 1 M_{\odot}$ stars (one of them being the HH 111 source), in a circular orbit with a separation of $\sim 186 \mathrm{AU}$, would produce the mirror symmetric pattern seen in the outflow.
\end{abstract}

Key words: circumstellar matter - Herbig-Haro objects - infrared: ISM - ISM: individual objects (HH 111) ISM: jets and outflows - stars: formation

Online-only material: color figure

\section{INTRODUCTION}

Over the past two decades considerable progress has been made in understanding the formation of stellar jets and their influence on the surrounding interstellar medium (see, e.g., Bally et al. 2007; Arce et al. 2007). Nevertheless, some fundamental questions remain unanswered, mostly related with the nature of the acceleration and collimation of the jets themselves, the properties of the "launch region," and how both of these processes can be influenced by nearby objects, since many outflows arise from binary or multiple young stellar systems.

Since the discovery of Herbig-Haro (HH) 111 (Reipurth 1989) using standard narrowband imaging techniques to map the outflow in $\mathrm{H} \alpha$ and [S II] $\lambda \lambda$ 6717/6731, HH 111 has become an icon of highly collimated stellar outflows, and has been the subject of a number of studies across the entire wavelength spectrum to determine the proper motions of their "knots" (Hartigan et al. 2001; Coppin et al. 1998), kinematics (Reipurth et al. 1992; Raga et al. 2002a), ionization structure (Reipurth 1989; Davis et al. 2001; Nisini et al. 2002), circumstellar environment (Stapelfeldt \& Scoville 1993; Yang et al. 1997), shock properties (Noriega-Crespo et al. 1993; Morse et al. 1993), molecular flow morphology (Cernicharo \& Reipurth 1996; Lefloch et al. 2007), and ejection history (Masciadri et al. 2002; Raga et al. 2002b), among others. The original optical images were of the West lobe of HH 111, and the Eastern counterjet was later identified in the near-infrared (NIR; Gredel \& Reipurth 1994; Davis et al. 1994).

HH 111 lies in the L1617 cloud in Orion at a distance of $\sim 417 \mathrm{pc}$ and is oriented almost E-W (P.A. $\sim 97.5)$ with a length of $\sim 11^{\prime}$ in our Infrared Array Camera (IRAC) images (see Figure 1). HH 111 is part of a quadrupolar flow (Reipurth et al. 1999; Rodríguez et al. 2008), with HH 121 arising nearly perpendicular (P.A. 27.3) to the HH 111 flow, suggesting that the VLA1 source is part of a close binary system. Reipurth et al. (1999) proposed that this binary could have a separation of $\sim 0$ '. 1 ( $\sim 50$ AU). Rodríguez et al. (2008) detect two elongated structures with a separation of $\sim 0^{\prime} 036(\sim 15 \mathrm{AU})$, which could correspond to the previously proposed binary.
Finally, we should note that $\mathrm{HH} 111$ is the central region of a "giant $\mathrm{HH}$ jet," with two lobes extending out to $\approx 3.5 \mathrm{pc}$ from the outflow source (Reipurth et al. 1997). The far ends of these lobes (HH 311 and HH 113; see Reipurth et al. 1997) show a point-symmetric deviation from the direction of the inner HH 111 outflow of $\approx 4^{\circ}$. This change in direction between the inner and outer regions of the HH 111 outflow is evidence for a precession of the outflow axis, with a period $\tau_{p} \approx$ $3.5 \mathrm{pc} / 150 \mathrm{~km} \mathrm{~s}^{-1} \approx 23,000 \mathrm{yr}$ (considering the proper motion of $\sim 150 \mathrm{~km} \mathrm{~s}^{-1}$ measured by Reipurth et al. 1997 for HH 311 and 113).

Recently, we have shown (Raga et al. 2011) that by using observations from the Spitzer Space Telescope (Spitzer; Werner et al. 2004) in the mid-infrared, where the extinction is considerably lower than in the optical and NIR, one can use the jet/counterjet symmetry to set some of the strongest constraints yet on the size of the jet formation region. In this study, we take advantage once again of the superb sensitivity and angular resolution of the IRAC (Fazio et al. 2004) on board of Spitzer to study the positional distribution of the "knots" along the HH 111 flow, finding mirror symmetric deviations from the jet/counterjet axis. This is the first time that such a mirror symmetric pattern is seen in an $\mathrm{HH}$ outflow, which can be interpreted in terms of the presence of a binary outflow source.

\section{DATA}

The observations of HH 111 are part of our original Spitzer General Observer program 3315 (PI: Noriega-Crespo) obtained with IRAC and the infrared photometer MIPS (Rieke et al. 2004) in 2005 March 28. The data have been downloaded from the Spitzer Legacy Archive and the quality of the final images (post basic calibrated data or post-BCD; S18.7 products) is outstanding, so that no further processing was required. The HH 111 outflow is part of a larger mosaic of the L1640 region obtained with IRAC in its four channels $(1,2,3$, and 4$)=$ (3.6, 4.5, 5.8, and $8.0 \mu \mathrm{m})$ covering a field of view of $\sim 19 \times 23^{\prime}$ (the result of a $5 \times 4$ array map with a $260^{\prime \prime}$ stepsize) 


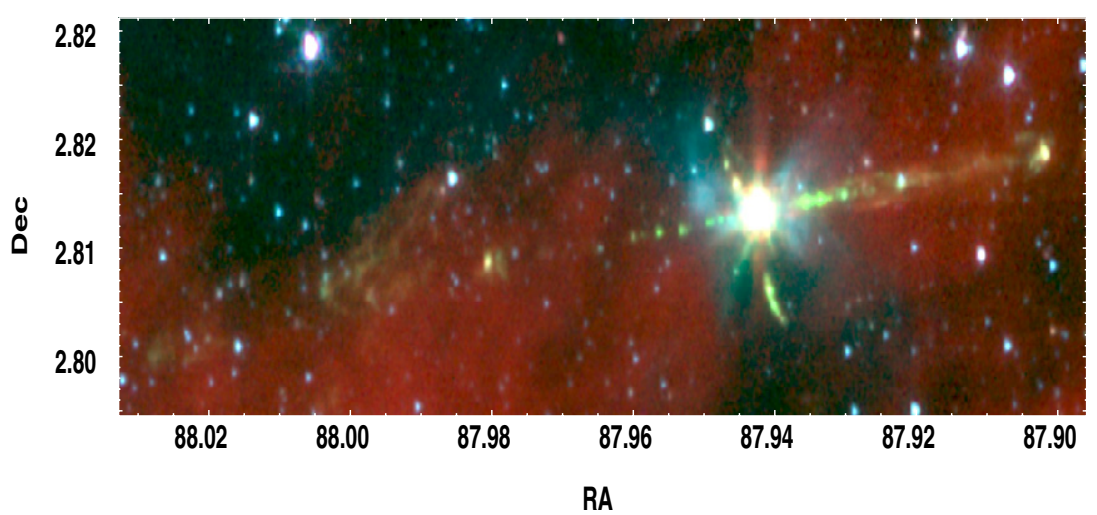

Figure 1. HH 111 flow in the mid-IR using the 3.6 (blue), 4.5 (green), and $8 \mu \mathrm{m}$ (red) IRAC channels. Note the scattered light (blue) near the source and the nearly perpendicular bipolar outflow HH 121.

(A color version of this figure is available in the online journal.)

and with a total integration time per pixel of $360 \mathrm{~s}$, using $30 \mathrm{~s}$ High Dynamic Range exposures. The final images are sampled with 0 .' 6 pixel $^{-1}$, nearly one-third of the standard $\sim 2^{\prime \prime}$ IRAC angular resolution.

The IRAC images (Figure 1), in comparison with the published NIR images (e.g., Davis et al. 1994, Figure 5), display a similar morphology, but given their higher signal-to-noise and lower extinction permit us to define better the knots and identify a couple more on each side close to the VLA1 source. Like in other protostellar outflows observed with Spitzer (e.g., NoriegaCrespo et al. 2004; Looney et al. 2007; Tobin et al. 2007; Ybarra \& Lada 2009; Raga et al. 2011) HH 111 is brighter in channel 2 , although it is well detected in all four IRAC channels. The emission is most likely dominated by the $\mathrm{H}_{2}$ pure rotational lines (De Buizer \& Vacca 2010).

In the following we describe the emission peaks as condensations and/or knots. It is clear from the Hubble Space Telescope images at high angular resolution that many of these structures are bowshock-like (working surfaces) and that they can overlap one another. We use the intensity peaks to define the HH 111 flow. The measurements of the positions of the condensations/ knots were carried out using the IRAF imexam routine interactively on the IRAC channel 2 image. For compact "knots" a two-dimensional Gaussian was used for determining their centroids; for the more diffuse structures, we use the position of the pixel with the peak emission. The uncertainty of the centroids ranges from 0.1 to 0.3 of a fraction of a pixel for the bright and fainter knots, respectively. Since the "knots" are compact in most cases, we measured the flux densities using a circular aperture and an aperture correction. For the compact "knots" we used a 2".4 radius for the photometric measurements with a 1.221 aperture correction; the background was measured off the jet/counterjet flow axis. For seven "knots" that were obviously more extended or irregular than this aperture $\left(6.23 \times 10^{-10}\right.$ steradian area), a comparable polygonal area was used without an aperture correction. Some of these "knots" do have a low surface brightness, but it was still possible to measure the flux density and centroid.

In Table 1, we give the relative positions in $X$ (along the outflow axis) and $Y$ (perpendicular to the outflow axis) with respect to the central source, VLA1 at R.A. $=05: 51: 46.25$ and decl. = 02:48:29.5 (Reipurth et al. 1999) of the "knots" seen along the jet and the counterjet. Table 1 also contains their R.A. and decl. coordinates, flux density, and uncertainty (both in $\mathrm{mJy}$ ).
Table 1

Counterjet (East) and Jet (West) Positions and Flux Densities

\begin{tabular}{|c|c|c|c|c|c|}
\hline$X_{\text {off }}\left({ }^{\prime \prime}\right)$ & $Y_{\text {off }}\left({ }^{\prime \prime}\right)$ & R.A. $\left(^{\circ}\right)$ & Decl. $\left(^{\circ}\right)$ & $F(\mathrm{mJy})$ & Uncertainty (mJy) \\
\hline 0.00 & 0.00 & 87.9426 & 2.8084 & 123.89 & 12.39 \\
\hline-12.39 & -0.40 & 87.9460 & 2.8078 & 0.69 & 0.07 \\
\hline-18.25 & -0.14 & 87.9476 & 2.8076 & 0.46 & 0.05 \\
\hline-24.67 & 0.12 & 87.9494 & 2.8075 & 0.32 & 0.03 \\
\hline-38.78 & -0.75 & 87.9533 & 2.8067 & 0.18 & 0.02 \\
\hline-50.28 & -0.09 & 87.9564 & 2.8065 & 0.21 & 0.02 \\
\hline-58.53 & 0.60 & 87.9587 & 2.8064 & 0.06 & 0.01 \\
\hline-63.61 & 0.63 & 87.9601 & 2.8062 & 0.13 & 0.01 \\
\hline-105.40 & 0.39 & 87.9716 & 2.8045 & 0.02 & 0.01 \\
\hline-109.17 & 1.32 & 87.9727 & 2.8047 & 0.03 & 0.01 \\
\hline-132.28 & 2.92 & 87.9791 & 2.8042 & $0.37^{\mathrm{a}}$ & 0.04 \\
\hline-137.47 & 2.27 & 87.9805 & 2.8039 & $0.91^{\mathrm{b}}$ & 0.09 \\
\hline-199.16 & -1.37 & 87.9973 & 2.8006 & $0.22^{\mathrm{c}}$ & 0.02 \\
\hline-202.54 & 8.10 & 87.9986 & 2.8031 & 0.11 & 0.01 \\
\hline-208.84 & 8.47 & 88.0004 & 2.8029 & 0.11 & 0.01 \\
\hline-219.96 & 8.22 & 88.0034 & 2.8024 & 0.19 & 0.02 \\
\hline-221.45 & 3.17 & 88.0036 & 2.8010 & 0.21 & 0.02 \\
\hline-236.14 & -5.11 & 88.0074 & 2.7982 & 0.05 & 0.01 \\
\hline-293.33 & -9.45 & 88.0230 & 2.7948 & 0.06 & 0.01 \\
\hline-309.59 & -6.21 & 88.0275 & 2.7951 & 0.09 & 0.01 \\
\hline 0.00 & 0.00 & 87.9426 & 2.8084 & 123.89 & 12.39 \\
\hline 17.12 & 0.14 & 87.9379 & 2.8090 & 0.62 & 0.06 \\
\hline 26.18 & -0.13 & 87.9354 & 2.8093 & 2.82 & 0.28 \\
\hline 32.60 & -0.23 & 87.9336 & 2.8095 & 2.50 & 0.25 \\
\hline 44.99 & -0.45 & 87.9302 & 2.8099 & 0.67 & 0.07 \\
\hline 50.34 & -0.33 & 87.9288 & 2.8101 & 0.20 & 0.02 \\
\hline 54.88 & 1.25 & 87.9276 & 2.8107 & 0.18 & 0.02 \\
\hline 59.41 & -0.50 & 87.9263 & 2.8104 & 0.16 & 0.02 \\
\hline 74.28 & 0.20 & 87.9222 & 2.8112 & $1.61^{\mathrm{d}}$ & 0.16 \\
\hline 86.57 & 0.08 & 87.9188 & 2.8116 & 0.14 & 0.01 \\
\hline 112.24 & 0.86 & 87.9118 & 2.8128 & 0.10 & 0.01 \\
\hline 116.44 & 2.22 & 87.9107 & 2.8133 & 0.10 & 0.01 \\
\hline 129.70 & -0.68 & 87.9069 & 2.8130 & 0.09 & 0.01 \\
\hline 144.64 & -0.49 & 87.9028 & 2.8136 & $0.71^{\mathrm{e}}$ & 0.07 \\
\hline 147.73 & -0.43 & 87.9020 & 2.8137 & 1.77 & 0.18 \\
\hline 167.50 & -2.07 & 87.8965 & 2.8140 & $0.07^{\mathrm{f}}$ & 0.01 \\
\hline 226.05 & -5.10 & 87.8803 & 2.8154 & $0.04^{\mathrm{g}}$ & 0.01 \\
\hline
\end{tabular}

Notes.

a Over a $1.058 \times 10^{-10}$ steradian area.

b Over a $3.976 \times 10^{-10}$ steradian area.

c Over a $6.431 \times 10^{-10}$ steradian area.

${ }^{\mathrm{d}}$ Over a $8.292 \times 10^{-10}$ steradian area.

e Over a $6.685 \times 10^{-10}$ steradian area.

${ }^{f}$ Over a $7.615 \times 10^{-10}$ steradian area.

g Over a $8.377 \times 10^{-10}$ steradian area. 

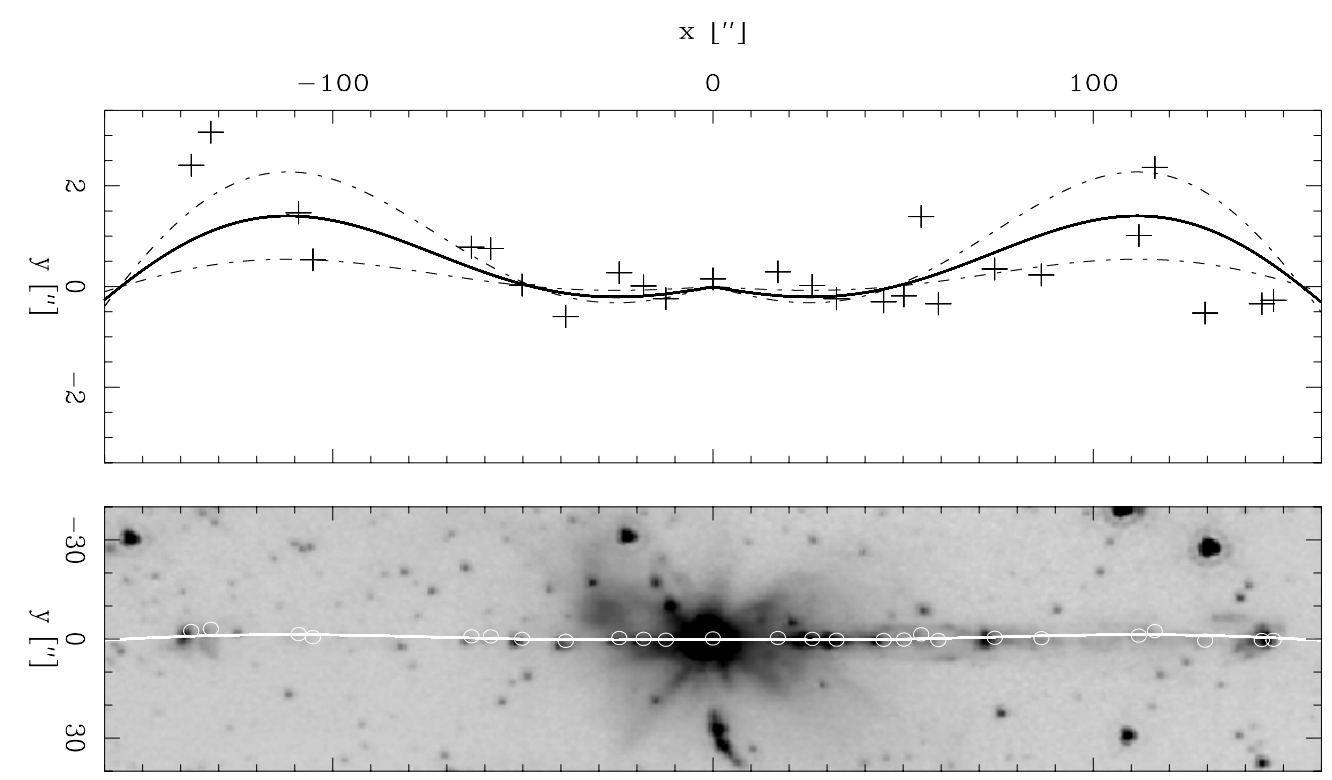

Figure 2. Right panel shows the central region of HH 111 at $4.5 \mu \mathrm{m}$ rotated so that its flow axis is vertical, and with the positions of the knots are shown with the white circles. The left panel shows the same knots positions (shown with crosses) with an expanded $Y$-axis (perpendicular to the outflow direction). The solid curve corresponds to a $\kappa=0.013$ ballistic jet (best-fit model), and the dashed lines correspond to $\kappa=0.005$ and 0.021 (the three models have $L=216^{\prime \prime}$ and $\phi=101^{\circ}$; see the text). The jet path predicted from the best-fit model is also shown on the right-hand side plot (white curve).

\section{THE MIRROR SYMMETRY OF THE HH 111 JET/COUNTERJET SYSTEM}

Figure 2 shows the inner region of the HH 111 outflow (top) with the measured $(X, Y)$ positions of the knots with respect to the VLA1 source. The bottom plot shows the knot positions with an expanded $Y$-coordinate. It is clear that both the jet and the counterjet show an excursion in the $-Y$-direction at a distance of $\approx 40^{\prime \prime}$ from the source, and an excursion in the $+Y$-direction at $\approx 115^{\prime \prime}$ from the source. These excursions could be part of a mirror symmetric spiral shape with a step of $\sim 2 \times\left(115^{\prime \prime}-40^{\prime \prime}\right)=150^{\prime \prime}$.

These mirror-symmetric excursions of the jet/counterjet system could possibly be the effect of an orbital motion of the outflow source. Ballistic flows from sources in circular and elliptical orbits were described by Masciadri \& Raga (2002) and González \& Raga (2004), respectively.

For a ballistic flow, the path of the beam of a constant velocity jet ejected parallel to the orbital axis (of an outflow source in a circular orbit) is given by

$$
\begin{aligned}
& y=\kappa x \sin \left(\frac{2 \pi}{\tau_{o} v_{j}} x-\psi\right), \\
& z=\kappa x \cos \left(\frac{2 \pi}{\tau_{o} v_{j}} x-\psi\right),
\end{aligned}
$$

where $x$ is the axial coordinate, $(y, z)$ are axes parallel to the orbital plane, $\psi$ is the orbital phase, $\tau_{o}$ is the orbital period, and $\kappa=v_{o} / v_{j}$ is the ratio between the orbital and the jet velocities. As can be seen from a comparison of Equations (1) and (2) with the results presented by Masciadri \& Raga (2002), in these equations we have considered that the orbital radius is negligibly small compared to $y$ and $z$.

From Equations (1) and (2), we see that the spiral described by the jet beam has a step

$$
L=v_{j} \tau_{o}
$$

From Equation (1), we calculate the predicted locus of the jet/counterjet structure and project it onto the plane of the sky, assuming an angle of $10^{\circ}$ between the orbital axis and the plane of the sky (Reipurth et al. 1992), and that the $y$-axis is parallel to the plane of the sky. We then carry out a least-squares fit in order to obtain the values of the model parameters that best fit the $\mathrm{HH}$ 111 jet/counterjet locus within $150^{\prime \prime}$ from the outflow source. The results of the fit are shown in the left panel of Figure 2.

The following parameters are obtained from the fit.

1. An orbital phase $\phi=(101 \pm 38)^{\circ}$. This result implies that the line joining the two stars of the binary outflow source currently lies close to the line of sight.

2. A spiral step $L=(216 \pm 39)^{\prime \prime}$. For a distance of $414 \mathrm{pc}$ to $\mathrm{HH} 111$ this corresponds to a distance of $(1.34 \pm$ $0.24) \times 10^{18} \mathrm{~cm}$. Also, from the proper motions measured by Hartigan et al. (2001), we see that the knots at $\sim 40^{\prime \prime}$ along the jet (corresponding to the first sideways excursion in the jet path; see Figure 2) have a spatial velocity $v_{j} \approx 240 \mathrm{~km} \mathrm{~s}^{-1}$. From Equation (3) we then obtain an orbital period $\tau_{o}=1801 \pm 317 \mathrm{yr}$.

3. A $\kappa=0.013 \pm 0.008$ (see Equations (1) and (2)). For a jet velocity of $240 \mathrm{~km} \mathrm{~s}^{-1}$ (see above), this implies an orbital velocity $v_{o}=3.1 \pm 1.9 \mathrm{~km} \mathrm{~s}^{-1}$.

We now use the relation (valid for a circular orbit) between the mass of the jet source $M_{1}$, the mass of the companion $M_{2}=\alpha M_{1}$, the orbital period $\tau_{o}$, and the orbital radius (of the jet source) $r_{o}$ :

$$
\frac{\alpha^{3} M_{1}}{(1+\alpha)^{2}}=\frac{\tau_{o} v_{o}^{3}}{2 \pi G}=(0.10,2.02,6.97) M_{\odot},
$$

where the three values in the third term correspond to $(\kappa, L)=$ $\left(0.005,177^{\prime \prime}\right)$ (the lower limits for $\kappa$ and $L$, see above), $(\kappa, L)=$ $\left(0.013,216^{\prime \prime}\right)$ (the values that result in the lowest $\left.\Xi^{2}\right)$, and $(\kappa, L)=\left(0.021,255^{\prime \prime}\right)$ (the upper limits).

Each of the three values of the third term of Equation (4) corresponds to a curve in the $M_{2}$ versus $M_{1}$ plane, which are 


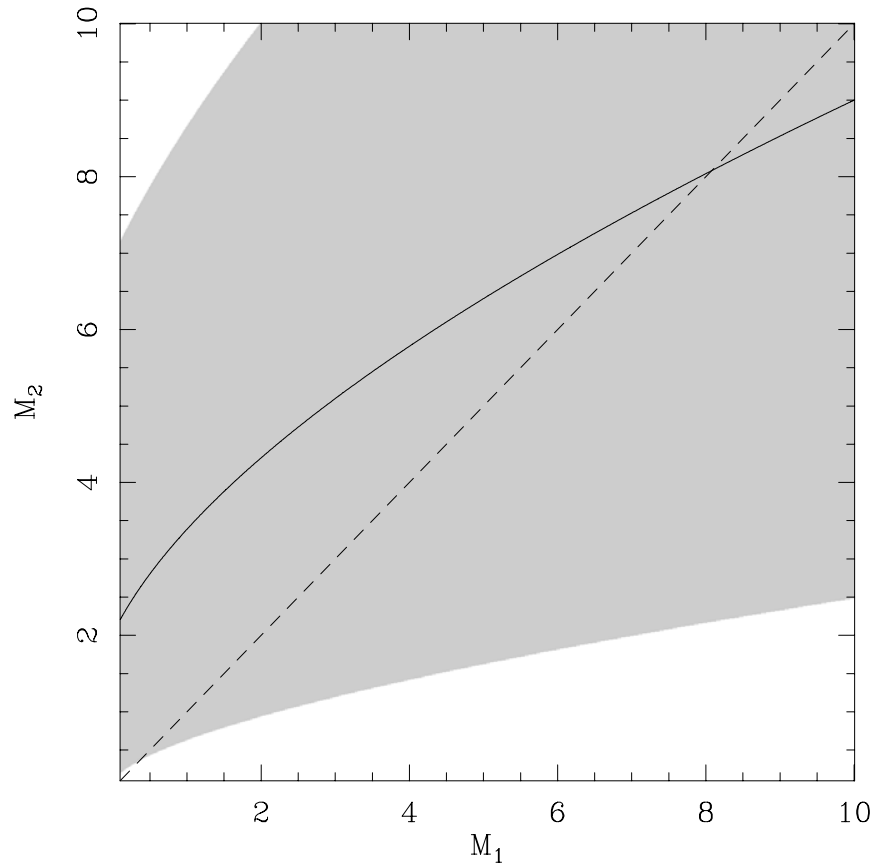

Figure 3. Parameter space for the primary and secondary masses of the binary system $\left(M_{1}\right.$ and $M_{2}$, in units of $\left.M_{\odot}\right)$ derived from the curved shape of the $\mathrm{HH} 111$ jet/counterjet system. The two curves represent the limits for the allowed masses derived from fitting the ballistic, orbiting source jet model to the observed trajectory (see Equation (4)). The shaded region shows the parameter space allowed by the fit. The solid curve is deduced from the parameters of the best fit. If one assumes that $M_{2}<M_{1}$ (i.e., that the source of the HH 111 outflow is the more massive star in the binary), the masses of the binary should lie beneath the $M_{2}=M_{1}$ line (the straight diagonal cutting across the plot).

shown in Figure 3. Together with the $M_{2}<M_{1}$ condition (i.e., assuming that the source of the strong $\mathrm{HH} 111$ outflow is the more massive star of the binary), we then obtain an allowed region for the masses of the $\mathrm{HH} 111$ source binary.

From Figure 3, we see that the observed shape of the HH 111 jet/counterjet system is consistent with a binary with two $\sim 1 M_{\odot}$ stars. Such a mass is consistent with the masses of T Tauri stars.

If we consider $M_{1}=M_{2}=M \approx 1 M_{\odot}$ (consistent with the curved shape of the HH 111 system; see Figures 2 and 3) and $\tau_{o}=1800 \mathrm{yr}$, from the equations of a circular orbit we then obtain a separation $2 r_{o} \approx 186$ AU between the two stars in the binary. Given that the orbital phase derived from the fit implies that the stars in the binary are closely aligned with the line of sight, the projected separation between the stars would be substantially smaller than $186 \mathrm{AU}$.

\section{SUMMARY AND CONCLUSIONS}

We have presented new IRAC images of the HH 111 outflow. These images show chains of aligned knots along the jet and the counterjet. We have carried out astrometric measurements of the positions of these knots and have also determined their fluxes in the IRAC $2(4.5 \mu \mathrm{m})$ channel.

The jet/counterjet knots within $\sim 2^{\prime}$ from the outflow source show mirror symmetric deviations from the outflow axis, which can be interpreted as the effect of an orbital motion of the outflow source. If we fit the observed shape of the projected path of HH 111 with a ballistic jet model (Masciadri \& Raga 2002), we find that the observations imply the existence of a binary with a $\tau_{o}=1801 \pm 317 \mathrm{yr}$ period and an orbital velocity $v_{o}=3.1 \pm 1.9 \mathrm{~km} \mathrm{~s}^{-1}$. This range of periods and orbital velocities would be consistent with a binary formed by two $\sim 1 M_{\odot}$ stars, with a separation of $\sim 186$ AU. The model fit also implies that the stars are currently quite closely aligned with the line of the sight, so that this separation might be consistent with the considerably lower projected separation implied by the observations of Rodríguez et al. (2008).

Interestingly, the orbital period that we determine $\left(\tau_{o} \approx\right.$ $1800 \mathrm{yr}$, see above) is $\sim 13$ times smaller than the precession period $\tau_{p}$ implied by the point-symmetrical "HH 111 giant jet" (see Reipurth et al. 1997 and the discussion at the end of Section 1). The resulting $\tau_{p} / \tau_{o} \sim 13$ ratio is consistent with the predictions from the precessing accretion disk models of Terquem et al. (1999).

Raga et al. (2009) pointed out that the models of Terquem et al. (1999) implied that outflows from binary sources would have mirror symmetries close to the outflow source (due to the orbital motion) and point symmetries at larger distances (if the ejection direction follows the disk precession). Our new observations show that the HH 111 jet/counterjet system has mirror symmetry within $\sim 2^{\prime}$ from the source. This result, combined with the point symmetry of the $\mathrm{HH} 111$ giant jet (Reipurth et al. 1997), is the first evidence ever of an astrophysical jet with the predicted flip in types of symmetry between regions close to and far away from the outflow source.

It is evident that the mirror symmetric, side-to-side excursions of the HH 111 jet/counterjet are a quite subtle effect. If our interpretation of these deviations were incorrect, we would not expect that a comparison with a model of an outflow from an orbiting source would give us reasonable masses for the binary components. However, we find that a binary with two $\sim 1 M_{\odot}$ stars and a separation of $\sim 200$ AU produce the observed spiral shape of HH 111. This completely reasonable result is a strong indication that we might indeed be interpreting the data correctly (see also the case for HH 211 by Lee et al. 2010).

This work is based in part on observations made with the Spitzer Space Telescope which is operated by the Jet Propulsion Laboratory, California Institute of Technology under NASA contract 1407. The work of A.R. and V.L. was supported by the CONACyT grants 61547, 101356, and 101975.

\section{REFERENCES}

Arce, H. G., et al. 2007, in Protostars and Planets V, ed. B. Reipurth, D. Jewitt, \& K. Keil (Tucson, AZ: Univ. Arizona Press), 245

Bally, J., Reipurth, J., \& Davis, C. J. 2007, in Protostars and Planets V, ed. B. Reipurth, D. Jewitt, \& K. Keil (Tuscon, AZ: Univ. Arizona Press), 215

Cernicharo, J., \& Reipurth, B. 1996, ApJ, 460, 57

Coppin, K. E. K., Davis, C. J., \& Micono, M. 1998, MNRAS, 301, 10

Davis, C. J., Hodapp, K. W., \& Desroches, L. 2001, A\&A, 337, 285

Davis, C. J., Mundt, R., \& Eislöffel, J. 1994, ApJ, 437, 55

De Buizer, J. M., \& Vacca, W. D. 2010, AJ, 140, 196

Fazio, G., et al. 2004, ApJS, 154, 10

González, R. F., \& Raga, A. C. 2004, RevMexAA, 40, 61

Gredel, R., \& Reipurth, B. 1994, A\&A, 289, 19

Hartigan, P., Morse, J. A., Reipurth, B., Heathcote, S., \& Bally, J. 2001, ApJ, 559, L157

Lee, Ch.-F., Hasegawa, T. I., Hirano, N., Palav, A., Shang, H., Ho, P. T. P., \& Zhang, Q. 2010, ApJ, 713, 731

Lefloch, B., Cernicharo, J., Reipurth, B., Pardo, J. R., \& Neri, R. 2007, ApJ, 658,498

Looney, L. W., Tobin, J. J., \& Kwon, W. 2007, ApJ, 670, 131

Masciadri, E., \& Raga, A. C. 2002, ApJ, 568, 733

Masciadri, E., Velázquez, P. F., Raga, A. C., Cantó, J., \& Noriega-Crespo, A. 2002, ApJ, 573, 260

Morse, J. A., et al. 1993, AJ, 106, 1139

Nisini, B., et al. 2002, A\&A, 393, 1035 
Noriega-Crespo, A., Garnavich, P. M., \& Raga, A. C. 1993, AJ, 106 1133

Noriega-Crespo, A., et al. 2004, ApJS, 154, 352

Noriega-Crespo, A., Garnavich, P. M., Moro-Martin, A., Reipurth, B., AlonsoHerreo, A., \& Heathcote, S. 2002, BAAS, 35, 567

Raga, A. C., Esquivel, A., Velázquez, P. F., Cantó, J., Haro-Corzo, S., Riera, A., \& Rodríguez-González, A. 2009, ApJ, 707, L6

Raga, A. C., Noriega-Crespo, A., Lora, V., Stapelfeldt, K. R., \& Carey, S. J. 2011, ApJ, 730, L17

Raga, A. C., Noriega-Crespo, A., Reipurth, B., Garnavich, P. M., Heathcote, S. Böhm, K. H., \& Curiel, S. 2002a, ApJ, 565, 29

Raga, A. C., Velázquez, P. F., Cantó, J., \& Masciadri, E. 2002b, A\&A, 395, 647

Reipurth, B. 1989, Nature, 340, 44

Reipurth, B., Raga, A. C., \& Heathcote, S. 1992, ApJ, 392, 145
Reipurth, B., Yu, K. Ch., Rodríguez, L. F., Heathcote, S., \& Bally, J. 1999, A\&A, 352, L83

Reipurth, B., et al. 1997, AJ, 114, 757

Rieke, G. H., et al. 2004, ApJS, 154, 25

Rodríguez, L. F., Torrelles, J. M., Anglada, G., \& Reipurth, B. 2008, AJ, 136 1852

Stapelfeldt, K. R., \& Scoville, N. Z. 1993, ApJ, 408, 239

Terquem, C., Eislöffel, J., Papaloizou, J. C. B., \& Nelson, R. P. 1999, ApJ, 512, L131

Tobin, J. J., Looney, L. W., Mundy, L. G., Kwon, W., \& Hamidouche, M 2007, ApJ, 659, 1404

Yang, J., Ohashi, N., Yan, J., Liu, C., Kaifu, N., \& Kimura, H. 1997, ApJ, 475, 683

Ybarra, J. E., \& Lada, E. A. 2009, ApJ, 695, 120

Werner, M. W., et al. 2004, ApJS, 154, 1 